\title{
Biological effects of EV71 infection in neural cells
}

\author{
Xia Cao" ${ }^{1 \#}$, Li-Chun Wang ${ }^{2 \#}$, Long-Ding Liü ${ }^{2}$, Yun Liao ${ }^{2}$, Cheng-Hong Dong ${ }^{2}$, Qi-Han Li $^{2 *}$ \\ ${ }^{1}$ Department of Immunology and Microbiology, Kunming Medical College, Kunming, China; ${ }^{\#}$ Both authors contributed equally to \\ this work \\ ${ }^{2}$ Institute of Medical Biology, Chinese Academy of Medical Sciences and Peking Union Medical College, Kunming, China; \\ **Corresponding Author: imbcams.lq@gmail.com
}

Received 23 May 2010; revised 25 June 2010; accepted 30 June 2010.

\begin{abstract}
Enterovirus 71 (EV71) can cause serious nervous system lesions but to date the pathogenesis has been unclear. Our results show that EV71 proliferates in the neural cells and leads to neural cell lesions. The study of the pathology of EV71 infection in neonatal rat brains shows that the invasive ability of EV71 to the nervous system in vivo may depend on many unknown factors.
\end{abstract}

Keywords: EV71; Neural Cell; Infection; Biological Effects

\section{INTRODUCTION}

EV71 shares many structural characteristics with Picorna virus and is also a member of the Enterovirus family. Additionally [1], EV71 has been defined as the major pathogen of the hand-foot and mouth disease (HFMD) [2]. The clinical features of HFMD caused by EV71 infection have drawn increased attention [3]. The clinical features of EV71 infection are different from other enteroviruses such as the CoxA16 and CoxB3 that also cause HFMD [4]. A number of severe clinical cases that occurred in an epidemic in the Taiwan and Fuyang regions in China $[5,6]$ suggest that EV71 infection may cause nervous system lesions and lead to a responsive heart and lung failure in some infected patients [7]. The clinical features indicate that the biological significance of EV71 infection in neural cells may affect the epidemiology and clinical consequences of HFMD. Thus, in addition to the study of the pathogenesis of EV71 infection and the epidemiological detection, the study of the biological effects of EV71 infection in neural cells is of

\footnotetext{
*Foundation item: This work supported by National Natural Science Funds for Young Scholar. (No. 30700205); supported by the Ph.D. Programs Foundation of Ministry of Education of China (No. 200600 23008).
}

great importance. A number of studies have reported the development of a mouse-adapted HEV71 strain and newborn mice infected with mouse-adapted HEV71 [8-10]. These evidence suggests that rodents are not resistant to EV71 infection. Etiological studies of EV71 have so far not provided a good explanation of how EV71 infects neural cells, mainly because there is no established neural cell tissue culture model. In our work, we used a primary rat nerve cell line to examine the basic biological characteristics of the process of EV71 infection in neural cells, as well as the pathological changes caused by EV71 infection in rats. Thus, a basic biological study of EV71 infection in neural cells is provided in this article.

\section{MATERIALS AND METHODS}

\subsection{Viruses and Cells}

The EV71-23 isolated strain was provided by the Dept. of Viral Immunology, the Institute of Medical Biology, Chinese Academy of Medical Sciences, which was reproduced and harvested in Vero cells with a titer of $10^{7} \mathrm{CCID} 50 / \mathrm{ml}$.

\subsection{Rat Cortex Neural Cell Cultures and Phenotype Identification}

Four SD neonatal rats were soaked in $75 \%$ cold ethanol for 3-5 min before being sacrificed by decapitation under sterile conditions. The rat brains were placed into precooling D-Hanks balanced salt solution. The cerebral cortex was separated by careful removal of the meninges and blood vessels and shredded into a size of about 1 $\mathrm{mm}^{3}$. The tissues were digested with $0.25 \%$ trypsin to a single cell suspension in DF12 complete medium containing $10 \%$ FBS, inoculated into culture bottles treated with PLL to a density of $1 \times 10^{6}$, and cultured in relative volume fractions in a $5 \% \mathrm{CO}_{2}$ incubator at $37^{\circ} \mathrm{C}$. After $12 \mathrm{~h}$ the original culture mediums were replaced with Neurobasal culture media containing 2\% B27 for maintenance culture, then one-half of the medium was replaced with Neurobasal/B27 every three days. Culture 
bottle coating methods: $0.01 \mathrm{mg} / \mathrm{ml}$ of PLL was used for coating for $1 \mathrm{~h}$ at $37^{\circ} \mathrm{C}$, and bottles were ready for inoculation after washing two times with sterile distilled water. After ten days, the culture medium was discarded, and the neurons were fixed in cold $4 \%$ paraformaldehyde for $20 \mathrm{~min}$. Next, $3 \% \mathrm{H}_{2} \mathrm{O}_{2}$ was added to block endogenous peroxidase. The cells were incubated with the primary anti-NSE polyclonal antibody $(1: 150)$ at $4{ }^{\circ} \mathrm{C}$ overnight, and then the secondary antibody (biotinlabeled goat $\mathrm{IgG}$ ) was added at room temperature for 30 min. The results were observed under an electron microscope after chromogenic freezing in DAB. In the negative control samples, the primary antibody was replaced with the $0.01 \mathrm{~mol} / \mathrm{L}$ PBS. All animal care and handling were performed in accordance with the guidelines specified by the Institutes of Health Guide for the Care and Use of Laboratory Animals of Yunnan. During the entire experiment, the animals were monitored online to avoid any unnecessary suffering and to ensure complete anaesthesia.

\subsection{Virus Titration}

Rat cortex neurons were cultured in 6-well plates for 10 days. The medium was aspirated, and $200 \mathrm{ul}$ of $10^{5} \mathrm{CC}$ ID50/ml virus was added to the cells for $1 \mathrm{~h}$ at $37^{\circ} \mathrm{C}$ for adsorption. Cell maintenance medium containing 1.0\% fetal calf serum was added and cells were incubated in $5 \% \mathrm{CO}_{2}$ at $37^{\circ} \mathrm{C}$. For the control group the cell maintenance media was added directly and cultured under the same conditions. The cells were harvested and diluted 10fold at $24 \mathrm{~h}$ and $48 \mathrm{~h}$. The infectious titer was determined to be 5.5 at $10^{-4}$ diluent degree by the micro-titration test.

\subsection{Immunofluorescence Analysis}

The coverslip of 10-day cultured cortex neurons $(1.0 \times$ $1.0 \mathrm{~cm}^{2}$ ) was washed lightly three times in cold 0.01 mol/L PBS ( $\mathrm{pH} 7.4$ ) and fixed with $4 \%$ paraformaldehyde for $30 \mathrm{~min}$. The fixed coverslip was washed three times with PBS, 5 min each time, incubated at room temperature for $10 \mathrm{~min}$ in $1 \% \mathrm{H}_{2} \mathrm{O}_{2}$, washed two times with PBS for $5 \mathrm{~min}$ each wash, and the coverslip was blocked with $1 \%$ bovine serum albumin at $37^{\circ} \mathrm{C}$ for 30 min. The bovine serum albumin was discarded, and anti-EV71-FY23 polyclonal mouse antibody (1:100) was added and incubated at $4{ }^{\circ} \mathrm{C}$ overnight. Next, the coverslip was washed three times with PBS and incubated with FITC labeled goat anti-mouse $\operatorname{IgG}(1: 300)$ for 2 hours. Finally, the coverslip was washed with PBS three times and photographed under a fluorescence microscope. In the negative control group, the primary antibody was replaced with $0.01 \mathrm{~mol} / \mathrm{L}$ PBS.

\subsection{Electron Microscopy of Infected Cells}

The cells were collected after digestion and separation with trypsin 24 hours after virus inoculation and placed into electron microscopy fixing solution mixed with $3 \%$ glutaraldehyde plus 1.5\% paraformaldehyde, followed by fixation with $1 \%$ osmium tetroxide, dehydration with gradient alcohol and embedding with epoxy resin. The neuron ultrastructure was observed through ultrathin sections under electron microscopy.

\subsection{Quantitative Detection of the EV71 Viral Load by Real-Time PCR}

Cells infected with EV71-23 at different were collected at varying time-points. RNA (TRI REAGENT-BD) was extracted by the Trizol method. Real-time PCR was performed with a one-step PrimescriptTM RT-PCR (TaKaRa) kit. The primer sequences for EV71-23 (gene sequence number is eu812515) are FW primer: 5'-ccacctggagcccctaagccag-3', RV primer: 5'-cattgataagcactcgcagg-3'; and probe primer: 5'-FAM-agcgcaggtttcagtgccattcat-TAMRA3-3'. A volume of 2 ul of total RNA template, $0.5 \mathrm{ul}$ of $10 \mathrm{uM}$ upstream and downstream primers, and $1 \mathrm{ul}$ of Taq man probe solution were used for the reaction for 40 cycles at $42^{\circ} \mathrm{C}$ for $5 \mathrm{~min}$, at $95^{\circ} \mathrm{C}$ for $10 \mathrm{~s}$, at $95^{\circ} \mathrm{C}$ for $5 \mathrm{~min}$. and $60^{\circ} \mathrm{C}$ for $40 \mathrm{~s}$.

\subsection{Pathological Detection of EV71 Infection in the Nervous System of Rats}

$0.4 \mu \mathrm{m}$ thick paraffin sections of the brain of neonatal rats infected by EV71 for five days were used to perform the high-pressure antigen retrieval after deparaffin and dehydration with a gradient ethanol. A volume of $50 \mu \mathrm{l}$ of the primary antibody (VEGF, HO-1 antibody) was added to each slice and incubated in $4^{\circ} \mathrm{C}$ overnight. The secondary enzyme-labeled rabbit anti-mouse conjugate antibody was incubated with each slice at room temperature. DAB showed color after 15 minutes. The slices were observed under a microscope for 3-5 minutes, differentiated with $0.1 \% \mathrm{HCl}$, washed with tap water to blue, dehydrated and dried with a gradient ethanol, transparencied with xylene, and sealed with neutral gum.

\section{RESULTS}

\subsection{The Primary Passage Culture of Rat Neural Cells and the Phenotype Identification}

The neural cortex of neonatal rats were collected, dispersed, screened and cultured in 6-well plates. After 10 days, the cells grew in a monolayer indicating that the networks between neurons are very rich, which is a feature of neurons. Some neural cells assembled into a group (Figure 1(a)). To confirm that the cells were neural cells, rather than supporting cells, an immunohisto- 


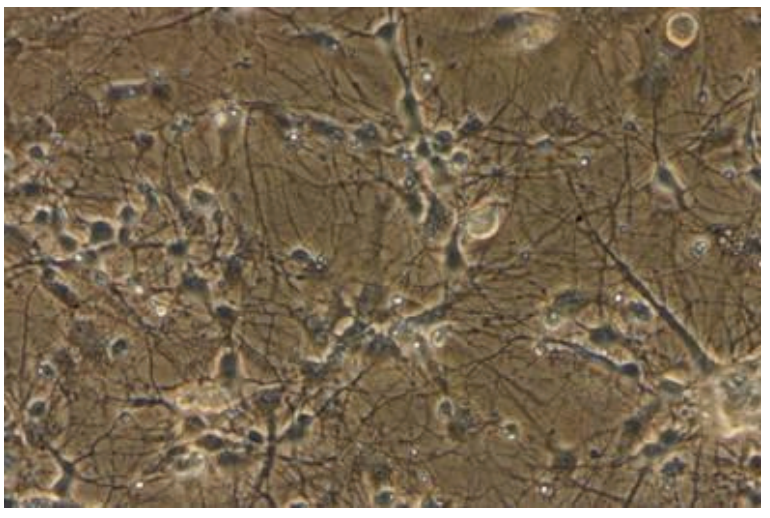

(a)

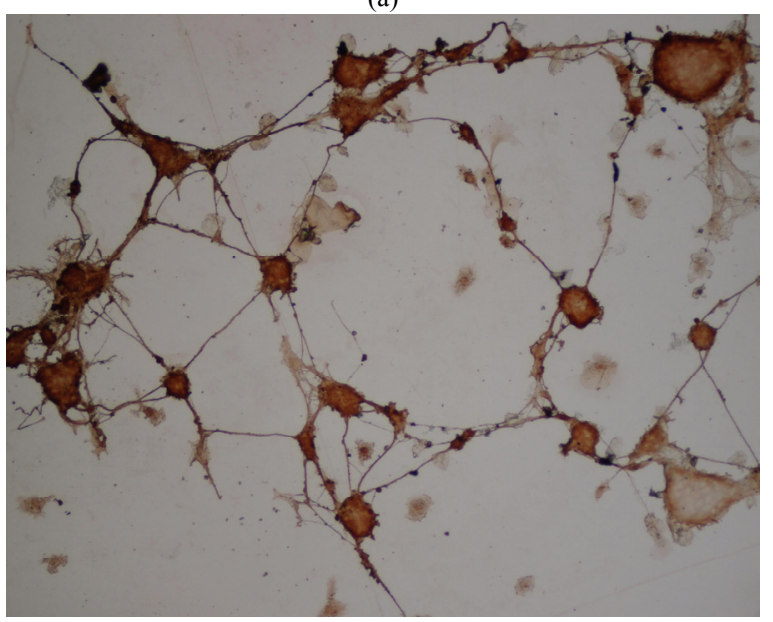

(b)

Figure 1. Primary neuron culture and phenotype identification of neonate rat. (a) Neuron at culture day $10(\times 200)$; (b) NSE labeled neuron $(\times 200)$.

chemistry staining test was performed using anti-NSE polyclonal antibody. The result showed that the cells had a clear neuronal phenotype (Figure 1(b)). Data indicate that neuron-specific enolase (NSE) is a specific marker of neuronal differentiation and maturation. NSE is a cytoplasmic protein and a dimer isozyme involved in the process of glycolysis, which is expressed mainly in neurons and located in neuronal cell bodies, axons and dendrites [11]. The chemical staining method using NSEspecific antibody to immune cells in our test identifies neurons in vitro and allows the analysis of the growth and development of neurons. Our cultured neural cells established a primary neural cell culture while maintaining their phenotypic characteristics.

\subsection{Infectious Proliferation of EV71 Cultured in Primary Neural Cells}

A monolayer of neural cells were inoculated with virus at an MOI of 0.01. Immunofluorescence analysis of neural cells that had been infected for 8 hours demonstrated EV71 antigen synthesis in the neural cells (Fig- ure 2(a)). Examination by light microscopy of neural cells that had been infected for 24 hours showed no pathological features but cells were observed to be round and shrinking (data not shown). However, the electron microscope examination of these cells demonstrated that EV71 infection of neuronal cells led to the typically observed cell lesions, cell degeneration and necrosis, and rupture of cell membrane. In addition, organelles with membrane structures in the cytoplasm were ruptured and dissolved (Figure 2(b)). There was no remarkable impact found in red blood cells.

\subsection{Analysis of the Growing Dynamics of Infectious Proliferation of EV71 in Infected Neural Cells}

Each virus has its own particular growth dynamics in susceptible cells. The proliferation of EV71 in other cells, such as Vero cells and RD cells, shows that its proliferation is similar to other enteroviruses [12]. An analysis of absolute amounts of viral proliferation by an infection at an MOI of 1 in $10^{4}$ neural cells showed that up to $10^{6} \mathrm{CCID} 50$ of virus were harvested in $24 \mathrm{~h}$ (Figure 3(a)). The amplification curve from RT-PCR data of EV71 infection in neural cells demonstrates that the proliferation peak occurred $12 \mathrm{~h}$ after infection and declined soon after (Figure 3(b)).

\subsection{Observation of the Infectious Pathology of EV71 in the Neonatal Rat Brain}

Although EV71 is able to infect neural cells under tissue culture conditions in vitro, its invasive ability of infection of the nervous system in vivo may depend on more unknown factors. Therefore, we further explored in vivo infection by inoculating EV71 into the nerve tissue of neonatal rats by intracerebral injection to analyze infection using clinical and histopathological indicators. Observation of neonatal rats inoculated with $10^{5} \mathrm{CCID} 50$ EV71 by intracerebral injection for 10 days indicated that EV71 did not cause the experimental neonatal rat group to develop clinical symptoms (data not shown). However, a histopathological observation of brain nerve tissue of a neonatal rat, which was killed on the fifth day of infection, indicated that the intracerebral injection of EV71 did cause an apparent inflammatory reaction, emergence of nerve glial cell aggregation, and a small amount of nerve cell damage (Figures 4(a), (b), and (c)).

\section{DISCUSSION}

It is difficult to clinically distinguish HFMD caused by EV71 infection from CoxA16 infection but EV71 can cause nervous system lesions with higher mortality rates 

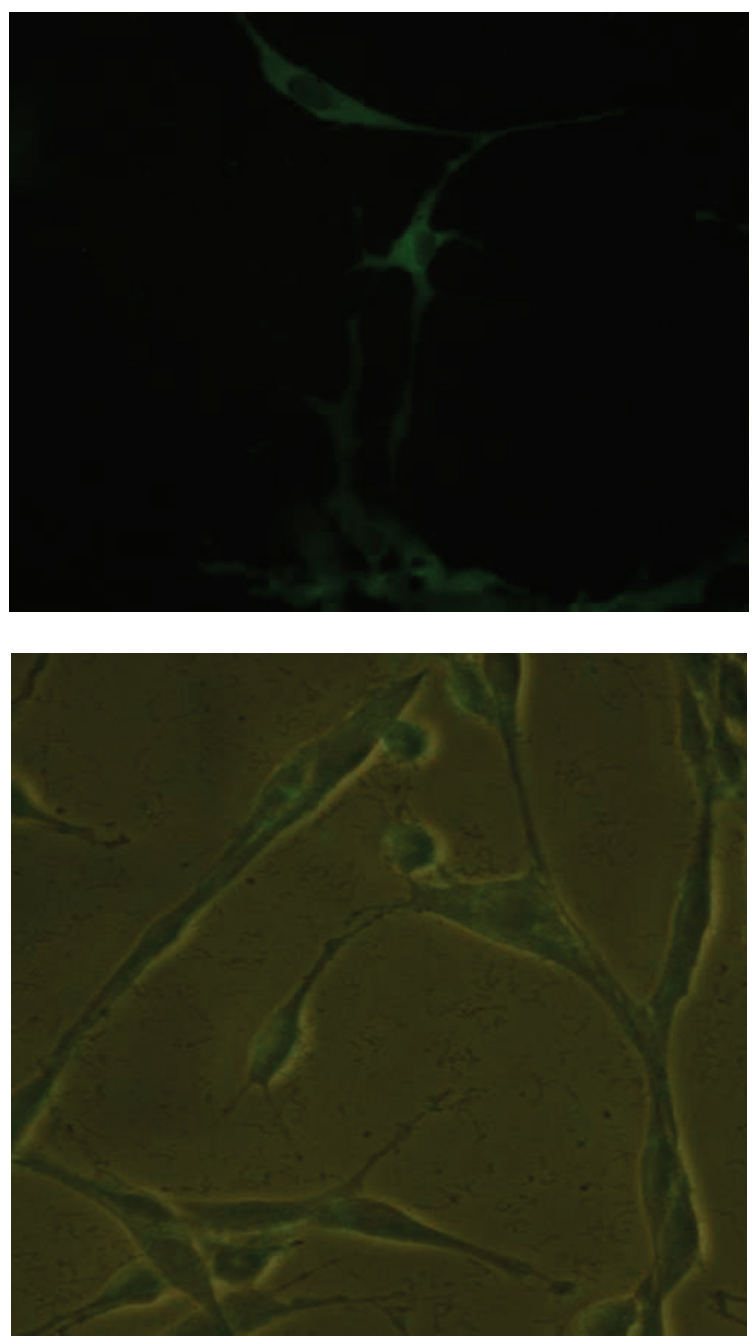

Negtive control

(a)

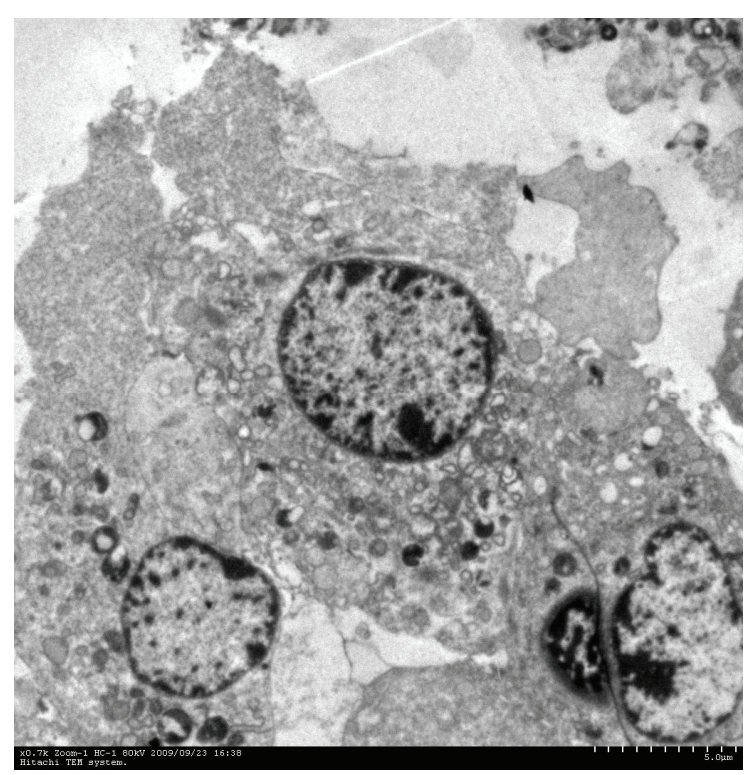

Copyright $\odot 2010$ SciRes.

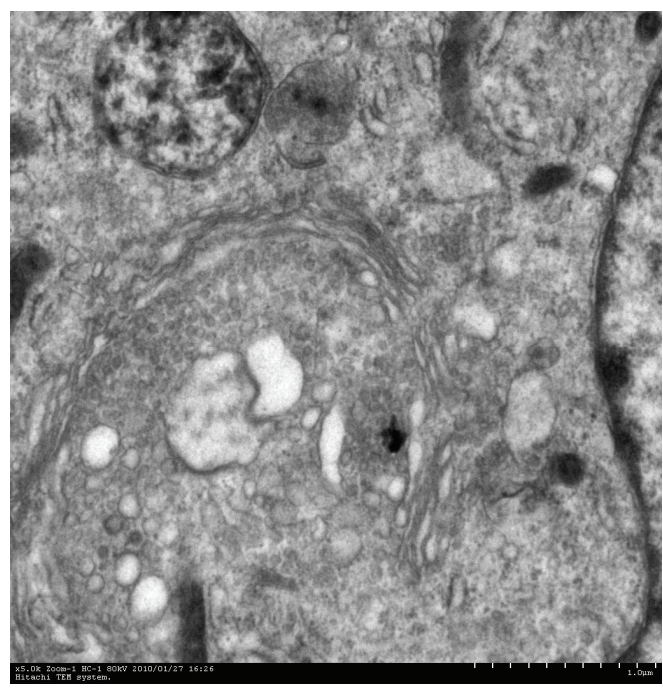

Negtive control

(b)

Figure 2. Cell biology change of EV71 infected neuron. (a) EV71 antigen synthesis in the neural cells; (b) Electron microscope examination of EV71 infected neuron.



(a)

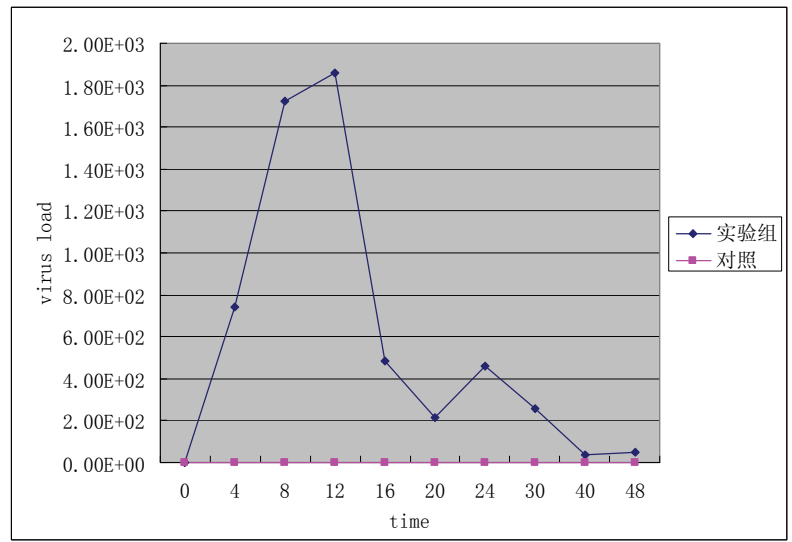

(b)

Figure 3. Growth kinetics analysis of EV71 infected neuron. (a) Proliferation of EV71 in neuron; (b) The amplification curve of EV71 infection in neuron.

Openly accessible at http://www.scirp.org/journal/JBPC/ 


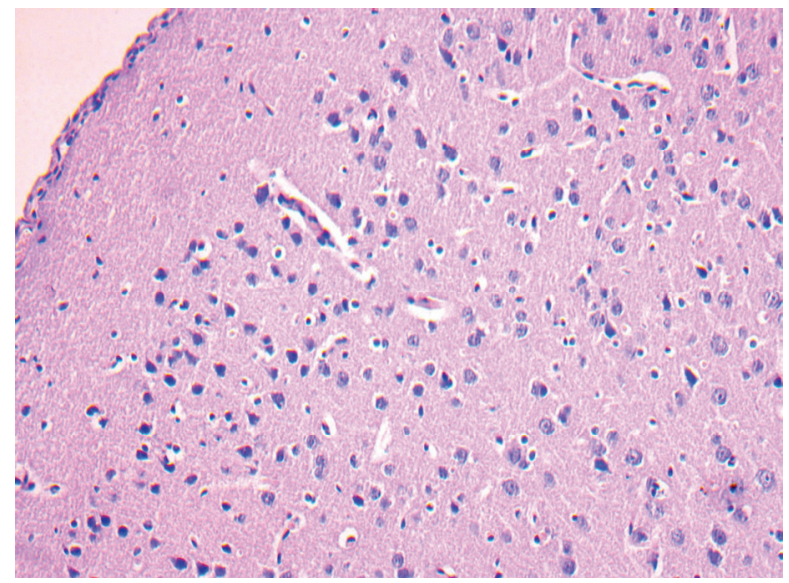

(a)

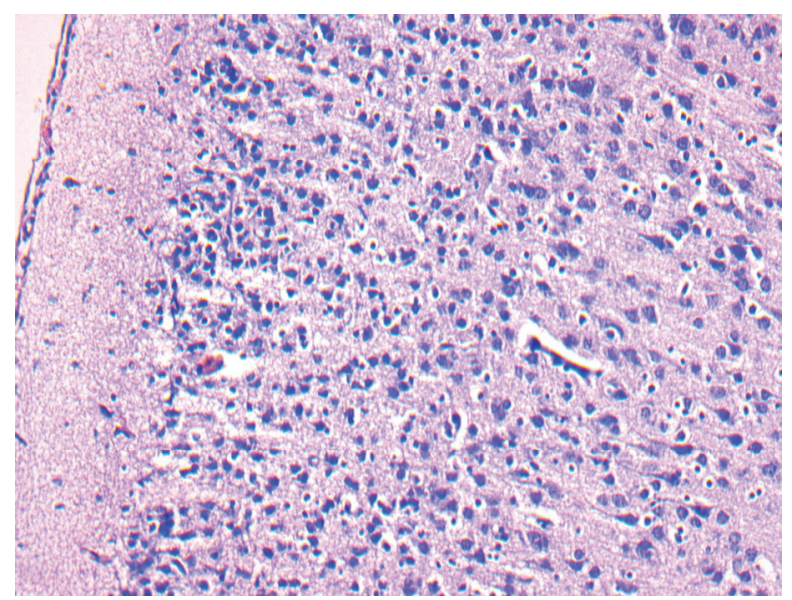

(b)

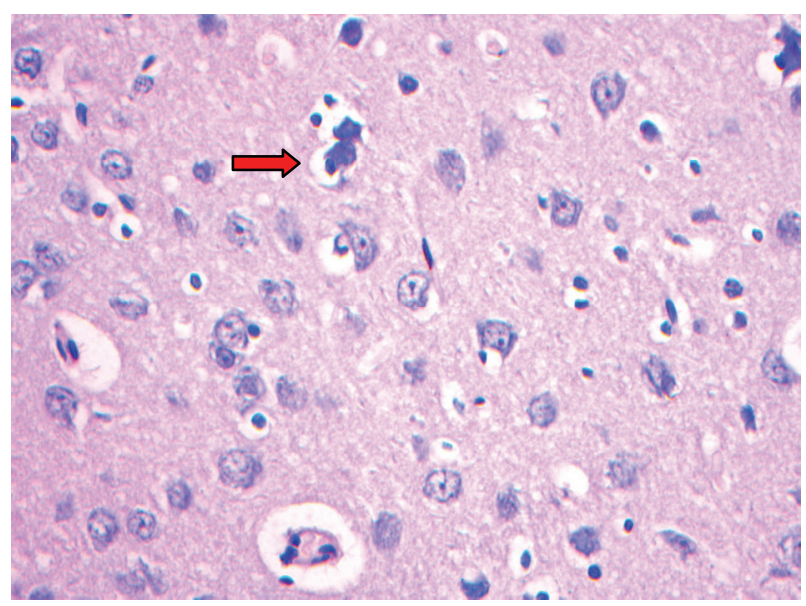

(c)

Figure 4. EV71 virus transcranial inoculates and infects neonate rats' nervous tissue. (a) Normal neonate rat $(\times 200)$; (b) Infected EV71 for 5 days $(\times 200)$, it is clear that glial cell diffusely hyperplasy; (c) Infected EV71 for 5 days $(\times 400)$ arrowhead showed neuronophagia.

and is an extreme hazard in children with severe infec- tions. However, extensive research on EV71 infection of the nervous system is lacking, and particularly there have been no studies in primary neural cells, which could have a great impact on understanding the epidemiology and clinical consequences of HFMD. Thus, analysis of the biological effects of EV71 infection in neural cells will help to understand the pathogenesis of EV71 infection and its clinical impact. As known before, HEV71 has a limited host range, with humans and some monkeys. Therefore, the high cost of monkeys and their maintenance precludes their widespread use for studies of viral pathogenesis. Thus, a small animal model would provide a more cost-effective and practical tool for studies of HEV71 pathogenesis and for vaccine development. A number of studies have suggested that rodents are not resistant to EV71 infection. Since we think mouseadapted EV71 strains are imperfect, so we want to search other cell lines which facility infected by EV71. On the other hand, it is more convenient to observe the pathological change in rat brain than that of mouse because the former is bigger that the latter. In our study, immunofluorescence analysis of neural cells infected for 8 hours showed EV71 antigen synthesis, which was concentrated in the cytoplasm and neural synapses. Although no lesions were observed under the optical microscope in neural cells infected for 24 hours, subsequent electron microscopy showed that organelles were dissolved and broken. The result showed that EV71 infection affected the cytoplasm and synapses, as observed by Immunofluorescence, as well as the nucleus. Electron microscopy also confirmed the role of EV71 in causing neural cells lesions. An analysis of the growth dynamics of EV71 during infection of neural cells showed specific growth dynamics associated with enterovirus proliferation, which demonstrates that neural cells are as susceptible to infection as other cells. These results show that EV71 could indeed infect and proliferate in neural cells in vivo. To further understand the role of EV71 infection in rats, we examined the pathology of infection in neonatal rat brains. A small amount of nerve cell damage, obvious inflammation and glial cell proliferation were found in the nerve tissue of neonatal rats that were infected for 5 days. This indicated that infection of neonatal rats resulted in a certain degree of central nervous system lesions. There were no obvious clinical symptoms observed among neonatal rats after 10 days infection. We speculate that this is because the nervous system lesions were mild and were able to recover gradually. The result further indicates that there are a variety of complex factors involved in EV71 infection in neural cells in vivo. Therefore, complex pathways should be considered in further studies of the mechanism of EV71 infection of the nervous system. 


\section{REFERENCES}

[1] Brown, B.A. and Pallansch, M.A. (1995) Complete nucleotide sequence of enterovirus 71 is distinct from poliovirus. Virus Research, 39(2-3), 195-205.

[2] Chan, K.P., Goh, K.T., Chong, C.Y., et al. (2003) Epidemic hand, foot, and mouth disease caused by human enterovirus 71, Singapore. Emerging Infectious Diseases, 9(1), 78-85.

[3] McMinn, P.C. (2002) An overview of the evolution of enterovirus 71 and its clinical and public health significance. FEMS Microbiology Reviews, 26(1), 91-107.

[4] Ho, M. (2000) Enterovirus 71: The virus, its infections, and outbreaks. Journal of Microbiology, Immunology and Infection, 33(4), 205-216.

[5] Lin, T.Y., Twu, S.J., Ho, M.S., et al. (2003) Enterovirus 71 outbreaks, Taiwan: Occurrence and recognition. Emerging Infectious Diseases, 9(3), 291-293.

[6] Wan, J.F., Zhu, L.Y., Liu, H., et al. (2008) Analysis of EV71 epidemic situation in Fuyang. Anhui Medicine, in Chinese, 29(4), 344-345.

[7] Huang, C.C., Liu, C.C., Chang, Y.C., et al. (1999) Neurologic complications in children with enterovirus 71 infection. The New England Journal of Medicine, 341(13), 936-942.

[8] Chen, Y.C., Yu, C.K., Wang, Y.F., et al. (2004) A murine oral enterovirus 71 infection model with central nervous system involvement. Journal of General Virology, 85(1), 69-77.

[9] Chung, Y.C., Ho, M.S., Wu, J.C., et al. (2008) Immunization with virus-like particles of enterovirus 71 elicits potent immune responses and protects mice against lethal challenge. Vaccine, 26(15), 1855-1862.

[10] Tung, W.S., Bakar, S.A., Sekawi, Z., et al. (2007) DNA vaccine constructs against enterovirus 71 elicit immune response in mice. Genetic Vaccines and Therapy, 5(1), 6.

[11] Schmechel, D.E., Brightman, M.W. and Marangos, P.J. (1980) Neurons switch from non-neuronal enolase to neuron-specific enolase during differentiation. Brain Research, 190(1), 178-181.

[12] Yang, Z.F., Qing, S., Mo, Z.Y., et al. (2009) Detection of five different cells' sensitivities to enterovirus 71 using plaque forming assay World Journal of Gastroenterology, in Chinese, 17(6), 573-577. 